\title{
ASPHODELUS CERASIFERUS J. GAY Y A. VILLARSII VERLOT (ASPHODELACEAE): CARACTERIZACIÓN Y TIPIFICACIÓN
}

\author{
Zoila DÍAZ LIFANTE y Benito VALDÉS
}

RESUMEN. Asphodelus cerasiferus J. Gay y A. villarsii Verlot (Asphodelaceae): Caracterización y tipificación. Se tipifican y caracterizan morfológica, ecológica y corológicamente Asphodelus cerasiferus J. Gay y A. villarsii Verlot, como una primera contribución al reconocimiento de estas dos especies de la Sect. Asphodelus.

Palabras clave. Asphodelus, Asphodelaceae, Liliaceae s.l., morfología, tipificación, taxonomía.

ABSTRACT. Asphodelus cerasiferus J. Gay and A. villarsii Verlot (Asphodelaceae): Characterization and typification. A. cerasiferus J. Gay and A. villarsii Verlot are typified and characterized morphologically and ecogeographically, as a first contribution to the recognition of these two species of Sect. Asphodelus.

Key words. Asphodelus, Asphodelaceae, Liliaceae s.l., morphology, typification, taxonomy.

\section{INTRODUCCIÓN}

En el género Asphodelus (Asphodelaceae), la sect. Asphodelus reúne especies rizomatosas perennes de gran tamaño. Hasta mediados del siglo XIX sólo se conocían dos especies atribuibles a esta sección: A. ramosus L. y $A$. albus Miller. A partir de entonces fueron descritas numerosas especies, entre las que se encuentran A. cerasiferus J. Gay y A. villarsii Verlot, ambas con características morfológicas comunes que las diferencian del resto de las especies de la sección.

El primer autor que puso en evidencia de forma impresa la existencia de A. villarsii como grupo distinto a las especies previamen- te descritas fue Billot (1854: 298), quien, a partir de plantas cultivadas en el Jardín Botánico de Grenoble en 1853, realizó una comparación detallada carácter a carácter de lo que él suponía dos especies o variedades distintas, una procedente de las montañas calizas de los alrededores de Grenoble y otra de Lautaret (Hautes-Alpes), a las cuales no dió nombre. Ambas habían sido englobadas en $A$. ramosus y en $A$. albus por Villars (1787: 205) y Mutel (1830: 429, 1848: 623) en sendas floras del Delfinado.

Más tarde Gay (1857a: 127, 1857b: 610) describió A. cerasiferus, al que caracterizó por su tallo simple o ramificado, brácteas pálidas y cápsulas esferoideas grandes, de $15-20 \mathrm{~cm}$. 
Incluyó en la variabilidad de este taxón la posibilidad de que se presentaran brácteas oscuras en plantas que crecían a altitudes elevadas, como las de los alrededores de Grenoble, hecho que él explicó por efecto del frío (Gay, 1857a: 132).

Esta variabilidad fue considerada demasiado amplia por algunos autores como Verlot (in Billot, 1857: 108), quien opinaba que Gay había reunido bajo $A$. cerasiferus dos especies, según él, diferentes: "une espèce du mont Rachet et de Comboire près de Grenoble avec une espèce de Marseille et d'autres localitès du Midi». Denominó A. villarsii a la primera y la diferenció de $A$. cerasiferus por su «tige noire [errata: moins] rameuse et moins haute, une floraison un peu plus précoce (ici), des fleurs moins nombreuses, plus grandes, des bractées noirâtres et non fauves, des fruits ovales-oblongs moins gros et non sphériques, applatis sur le sommet». Por otra parte distinguió $A$. villarsii de las plantas de Lautaret $(A$. subalpinus Gren. \& Godron) por la inflorescencia más gruesa y flores y frutos de gran tamaño, éstos no aplicados sobre el eje.

Gay (1858: 250) consideró que las diferencias aportadas por Verlot no eran suficientemente estables para considerar a esas especies como diferentes. No obstante, afirmó que ya conocía la existencia de plantas con brácteas oscuras y frutos menos gruesos, a las que él reconoció como Asphodelus b intermedius, y a la que remitió $A$. villarsii como sinónimo.

Por su parte Jordan (1860: 734) aceptó A. villarsii, reconociéndolo como uno de los 5 grupos de especies que él definió dentro de la sect. Asphodelus. Posteriormente Jordan (1860) y Jordan \& Fourreau (1868: 123) describieron además $A$. fuscatus y $A$. collinus, especies que relacionaron con $A$. villarsii, y dividieron $A$. cerasiferus J. Gay en varias especies más, basándose en diversos caracteres.

No obstante, $A$. villarsii no ha sido aceptado por la mayoría de los autores, y las plantas con brácteas oscuras, inflorescencia sim- ple y fruto grande y globoso han sido incluidas unas veces en $A$. albus Miller (Coste, 1905: 347), y otras en A. cerasiferus, tanto las de la Península Ibérica (Willkomm, 1862: 204; Merino, 1909: 7; Argüelles et al., 1984: 11), como las del N de África (Maire, 1958: 30). Más recientemente Richardson \& Smythies (1978: 368) han establecido la combinación $A$. albus subsp. villarsii (Verlot ex Billot) I. B. K. Richardson \& Smythies, caracterizando, erróneamente, este taxón por sus brácteas «whitishscarious» (Richardson \& Smythies, 1980: 17).

Esta disparidad de opiniones indica la dificultad de interpretar la variabilidad de $A$. villarsii, lo que ha motivado que no haya sido reconocida hasta la fecha. El objetivo de este trabajo es abordar el problema del reconocimiento de A. villarsii y A. cerasiferus mediante una primera caracterización morfológica, ecológica y geográfica, llevándose a cabo asimismo la tipificación de ambos taxones.

\section{MATERIAL Y MÉTODOS}

Este estudio ha sido basado en material de A. cerasiferus y A. villarsii procedente del Mediterráneo occidental, conservado en los herbarios B, BC, BCF, C, ELVE, FI, G, GDA, GDAC, GE, JACA, K, L, LD, LEB, LISE, LISU, LOU, LY, MA, MGC, MPU, O, P, PI, SALA, SANT, SEV y STR, complementado por la observación directa en el campo de numerosas poblaciones de la Península Ibérica y NW de África.

\section{RESULTADOS Y DISCUSIÓN}

\section{Morfología, ecología y distribución.}

Estas especies están íntimamente relacionadas morfológicamente, cuando se les compara en el contexto general de la sect. Asphodelus. Ambas coinciden principalmente 


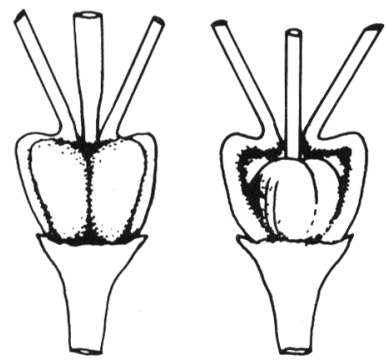

a
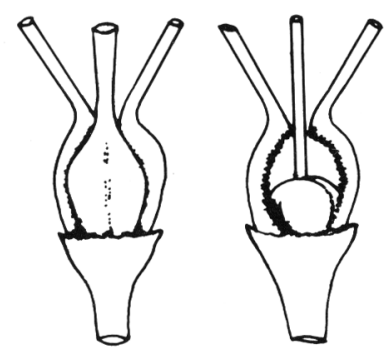

b

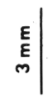

Figura 1. Base de los estambres y posición del ovario en Asphodelus cerasiferus J. Gay (a) y A. villarsii Verlot (b). Base of stamens and position of the ovary in Asphodelus cerasiferus J. Gay (a) and A. villarsii Verlot $(b)$.

en presentar frutos grandes y globosios, flores generalmente grandes y de tépalos anchos, brácteas anchas y pedicelos fructíferos gruesos. Sin embargo, presentan diversas diferencias morfológicas, que se indican en la tabla 1.

De esta tabla se deduce que $A$. cerasiferus y A. villarsii difieren fundamentalmente por los caracteres del rizoma, ramificación y densidad de las inflorescencias, color de las brácteas, longitud de los pedicelos y características de las bases ensanchadas de los filamentos estaminales (fig. 1).
En el Mediterráneo Occidental, $A$. cerasiferus se encuentra en el E de la Península Ibérica, S de Francia, Italia, Córcega, Cerdeña, E de Marruecos y $\mathrm{N}$ de Argelia. A. villarsii se encuentra en el $\mathrm{O}$ y $\mathrm{S}$ de la Península Ibérica, Alpes, Apeninos y NO de Marruecos (fig. 2). Por consiguiente las áreas de distribución de ambas especies son prácticamente alopátricas. A. cerasiferus se extiende por áreas de carácter típicamente mediterráneo, con escaso índice de pluviosidad, desde el nivel del mar hasta $1650 \mathrm{~m}$. A. villarsii ocupa

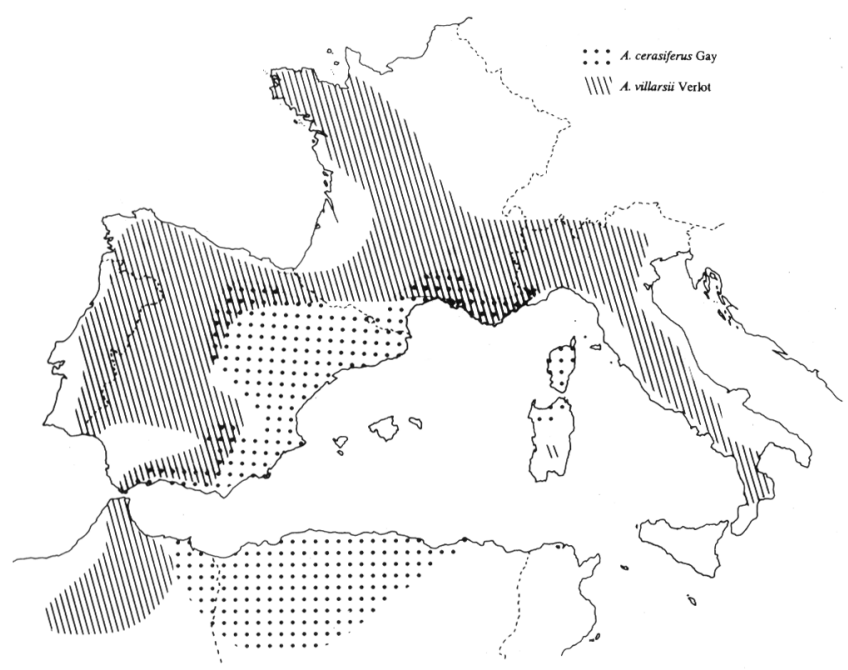

Figura 2. Distribución geográfica de Asphodelus cerasiferus J. Gay y A. villarsii Verlot en el Mediterráneo Occidental. Geographic distribution of Asphodelus cerasiferus J. Gay (dotted) and A. villarsii Verlot in the Western Mediterranean. 


CARACTERES Asphodelus cerasiferus J. Gay Asphodelus villarsii Verlot

Base de la roseta foliar

Tubérculos radicales

Hojas: disposición

Color de las hojas

membranosas

Escapo:
long. máxima
ramificación

\section{Brácteas}

Pedicelos:

long. en la flor

long. en el fruto

long. relativa a

las brácteas

articulación

Tépalos

Estambres:

base de los filamentos estaminales

Ovario

Estilo: longitud

Cápsula:

tamaño

forma

Semillas: tamaño generalmente rodeada de fibras finas desprovista de fibras o muy gruesas; a veces sin fibras

fusiformes, homogéneamente estrechados en los dos extremos; situados a $2-13 \mathrm{~cm}$ del rizoma

en espiral

blanquecinas o pardo-claras

$200 \mathrm{~cm}$

Con 1-5 (-9) ramas de hasta

$30(-40) \mathrm{cm}$, o simple

blanquecinas o pardo-claras, con negras o pardas

nervio medio pardo oscuro
6-16 (-19) mm

9-21 (-23) mm

más largos o más cortos

bien marcada

caducos

obovado-oblonga u oblonga, abruptamente estrechada en el ápice y anchamente surcada en el dorso (fig. 1a)

ocupando la cavidad formada por las bases de los estambres (fig. 1a)

$17-24 \mathrm{~mm}$

$10-20 \times 10-20 \mathrm{~mm}$

globosa; sección circular fusiformes, desigualmente estrechados en los dos extremos; situados próximos al rizoma o más raramente hasta $15 \mathrm{~cm}$ de éste

dística o en espiral

pardas o rojizas

$125(-185) \mathrm{cm}$

simple 0 , a veces, con 1-5 (-6) ramas de hasta $20(-35) \mathrm{cm}$

$6-13(-17) \mathrm{mm}$

6-17 (-19) mm

más cortos

a veces apenas marcada

caducos o persistentes en la

fructificación

lanceolada u oblongo-elíptica, gradualmente atenuada hacia el ápice, plana o con los márgenes ligeramente recurvados en la parte superior, particularmente en el verticilo externo (fig. 1b)

dejando un amplio espacio sobre él en la cavidad formada por las bases de los estambres fig. 1b)

$18-30(-32) \mathrm{mm}$

(9-) 12-18 (-19) x (9-) 12-18 (-20) mm globosa u elipsoideo-globosa; sección circular o más o menos triangular

$5-8,5 \times 2,5-4 \mathrm{~mm}$

Tabla 1. Caracteres morfológicos diferenciales de Asphodelus cerasiferus J. Gay y A. villarsii Verlot. Differential morphological characters of Asphodelus cerasiferus J. Gay and A. villarsii Verlot. 


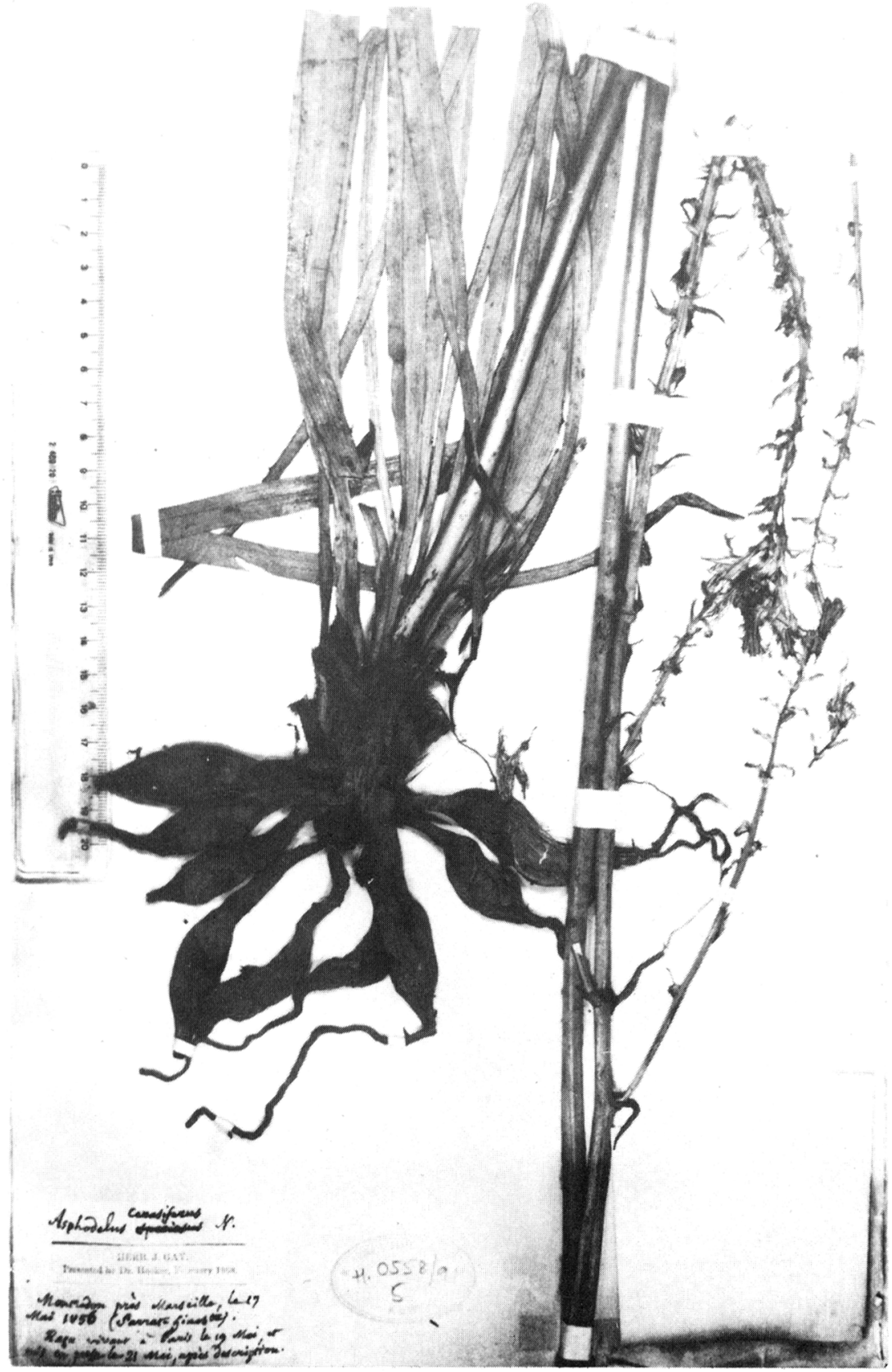

Figura 3. Lectotipo de Asphodelus cerasiferus J. Gay (K). Lectotype of Asphodelus cerasiferus J. Gay (K). 
las partes altas de los sistemas montañosos del Mediterráneo Occidental en zonas de carácter atlántico o mediterráneo, en este caso nunca por debajo de los $500 \mathrm{~m}$, y en general hasta los $2000 \mathrm{~m}$.

Tanto una como la otra crecen en ambientes expuestos y soleados, en pastizales, matorrales y claros de bosques (A. cerasiferus en pinares, encinares y coscojares, y A. villarsii en encinares, alcornocales, melojares, castañares y cedrales), sobre roquedos, laderas pedregosas y taludes, pero también a menudo en suelos de cierta profundidad. A. cerasiferus vive preferentemente sobre calizas, suelos margoso-yesosos, a veces de carácter aluvial, pero ocasionalmente también sobre pizarras, areniscas, serpentinas, e incluso rocas volcánicas. A. villarsii lo hace indistintamente tanto en suelos de carácter ácido, graníticos, pizarrosos o de areniscas, como calizos.

\section{Tipificación}

A. cerasiferus J. Gay, Ann. Sc. Nat., sér. 4, 7: 127 (1857).

- Ind. Loc. «dans la campagne de Montpellier».

- Lectotypus: ejemplar completo del pliego conservado en $\mathrm{K}$, en el que se leen las siguientes inscripciones (fig. 3):

-a [en etiqueta]: Asphodelus speciosus [tachado] cerasiferus N. // Montredon près Marseille, le 17 / Mai 1856 (Sarrat-Gineste). / Reçu vivant à Paris le 19 Mai, et / mis en presse le 21 Mai, après description. [ manuscr. Gay]. (fig. 4a).

- b [en etiqueta adherida a la etiqueta a]: HERB. J. GAY/ Presented by Dr. Hooker, February 1868 [impreso]. (fig. 4a)

-c [en etiqueta contenida en sobre]: Fleurs de l'Asphod. ... [tachado] cerasiferus .... Montredon / près Marseille [manuscr. Gay]. (fig. 4b).

- Comentarios: El lectotipo designado corresponde a un individuo completo, con rizoma cubierto por abundantes fibras delgadas, 9 tubérculos radicales fusiformes dispuestos a 2-3 $\mathrm{cm}$ del rizoma, con la porción proximal algo engrosada, hojas de hasta $40 \times 1,6 \mathrm{~cm}$ dispuestas en espiral, estrechadas hacia la base, escapo de c. $120 \mathrm{~cm}$, con 3 ramas cortas en la parte inferior, una de ellas separada, e inflorescencia laxa, con brácteas pálidas. Este ejemplar fue utilizado en la descripción de la especie, tal y como Gay indica en la etiqueta. La etiqueta $\mathrm{c}$ lleva adherida el extremo de una inflorescencia, y está contenida en un sobre de este mismo pliego, dentro del cual hay sueltas dos flores de $20 \times 3-5 \mathrm{~mm}$ y un fruto totalmente desarrollado de 18 x $18 \mathrm{~mm}$, con las valvas gruesas y los nervios poco marcados (fig. 4b).

Asimismo se han estudiado en el herbario de París (P) dos pliegos recolectados por Bourgeau en la provincia de Murcia, uno en Cartagena el 22 de Marzo de 1850 y otro en la Sierra de la Fuensanta el 28 de Marzo de 1852, los cuales fueron determinados por Gay como «A. cerasiferus Gay» el 11.IX.1857, y citados entre los materiales incluidos en la publicación de la especie (Gay, 1857a: 130), por lo que constituyen paralectotipos. Finalmente, se conserva en este mismo herbario un pliego procedente del herbario de Moquin-Tandon procedente de «Montpellier, près de Grabels», localidad también citada por Gay, cuyo ejemplar, determinado por él como "A. speciosus Gay» el 4.IX.1856, constituye igualmente un paralectotipo.

En la sesión extraordinaria de la Societé Botanique de France, que tuvo lugar en Montpellier en Junio de 1857, Gay leyó una comunicación titulada «Sur le distribution géographique de trois espèces de la Section Gamon du genre Asphodelus». Este trabajo fue publicado este mismo año en dos revistas diferentes casi al mismo tiempo: en el vol. 7 de Annales des Sciences Naturelles, 4 ser. (Gay, 1857a) y en el vol. 4 del Bulletin de la Societé Botanique de France (Gay, 1857b).

Según Leussink (1985:583), el $n^{\circ} 7$ del 


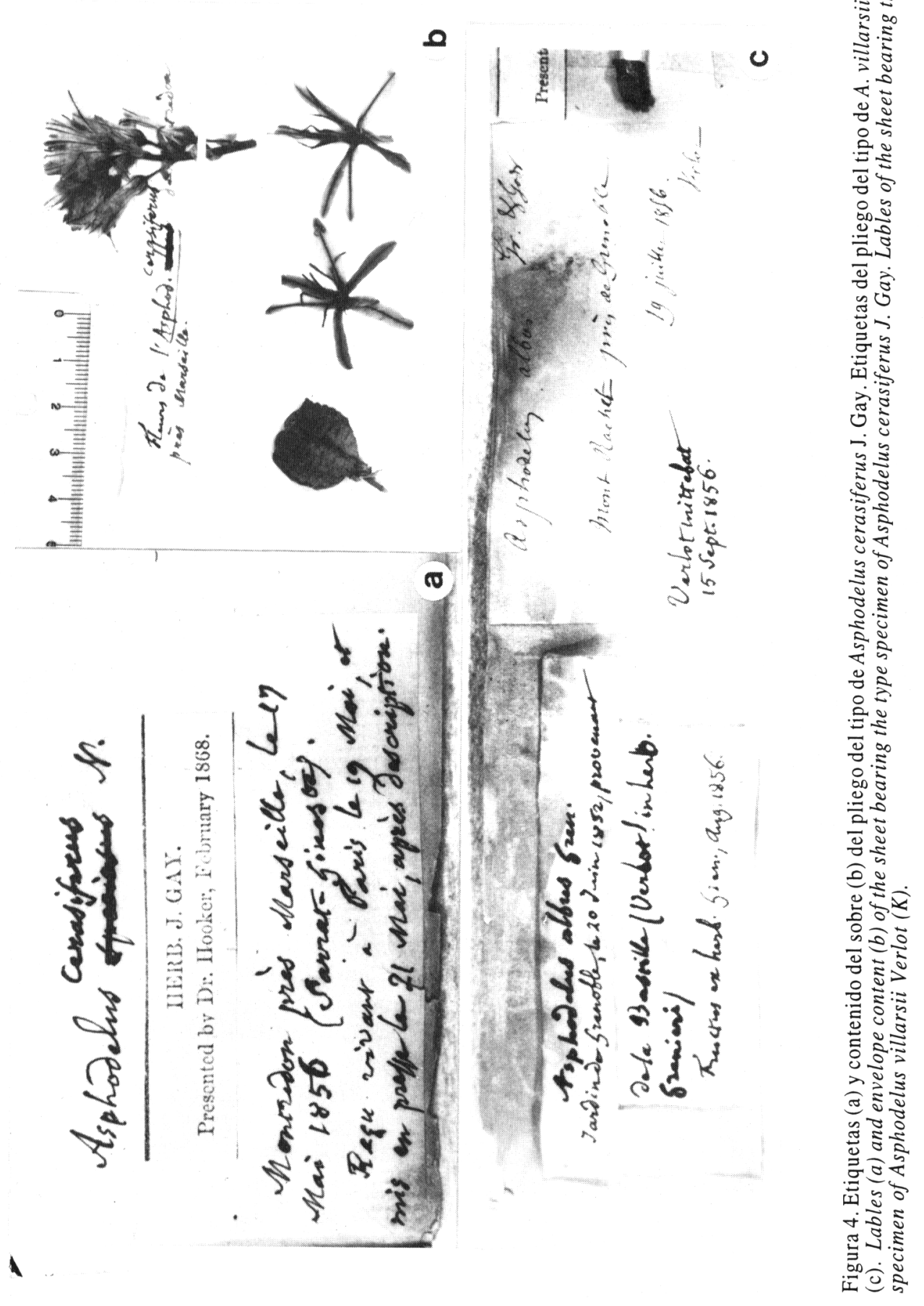


vol. 4 de Bull. Soc. Bot. Fr. no fue aprobado para su publicación por el Consejo de Administración de la Sociedad hasta el 11 de Septiembre de ese año y se publicó finalmente en Noviembre-Diciembre de 1857. Los volúmenes 7 y 8 de los Ann. Sc. Nat. (Bot), 4 sér., fueron publicados ambos en el mismo año, aunque cabe suponer que no simultáneamente, con lo que el volumen 7 saldría antes de finales de año. La publicación de los Annales, por otro lado más extensa, debe ser anterior a la del Bull. Soc. Bot. Fr., más resumida. El mismo Gay (1858: 252) al hacer referencia a su trabajo menciona primero los Annales, y después el Bull. Soc. Bot., y Billot (1857: 107) afirma que Verlot le comunicó el 19 de Noviembre de 1857 que había leído ya el trabajo de Gay en los Anales. Se considera por ello que el volumen 4 de los Anales constituye el lugar de publicación de la especie.

En cuando al nombre en sí, como ya señaló Maire (1958: 30), debe ser conservada su grafía original aunque el epíteto cerasifer, utilizado por Ascherson \& Graebner (1905: 33) y otros autores, sea más correcto.

A. villarsii Verlot in Billot, Annot. Fl. Fr. Allem. 108 (1857).

- Ind. Loc. «mont Rachet».

- Lectotypus: ejemplar con hojas y escapo, éste fragmentado, contenido en un pliego de $\mathrm{K}$ (fig. 5), en el que se leen las siguientes inscripciones:

-a [en etiqueta]: Asphodelus albus Gr. \& Godr. // Mont Rachet près de Grenoble // 19 juillet 1856 [manuscr. Verlot] // Verlot mittebat / 15 Sept. 1856 [manuscr. Gay].

-b [en etiqueta]: HERB. J. GAY / Presented by Dr. Hooker, February 1868 [impreso].

-c [en sobre]: Asphodelus albus Gren. / Jardin de Grenoble, le 20 Juin 1852, provenant / de la Bastille (Verlot! in herb. / Grenieri) / Fructus ex herb. Gren., Aug. 1856 [manuscr. Gay].
- Comentarios: En la carta que Verlot escribiera a Billot el 19 de Noviembre de 1857 (Billot, 1857: 108 y 109), se mencionan las localidades «mont Rachet» $\mathrm{y}$ "Comboire près de Grenoble» para referirse a las plantas que Gay confundió con A. cerasiferus de Marsella y otras localidades del «Midi» francés. Estas dos primeras localidades fueron también citadas posteriormente por Verlot (1860: 151, «montagnes calcaires autour de Grenoble telles que Comboire, Mont-Rachais, etc.») al tratar de esta especie. Se ha consultado material tipo de ambas localidades citadas, prefiriéndose utilizar en la tipificación de esta especie el ejemplar procedente de mont Rachet, ya que, según se lee en la carta anteriormente mencionada, Verlot basó las diferencias de A. villarsii con $A$. cerasiferus precisamente en la comparación de plantas cultivadas de $A$. villarsii procedentes de mont Rachet con plantas cultivadas de A. cerasiferus procedentes de Marse11 .

No se conserva ningún pliego de Verlot de $A$. villarsii en el herbario de Grenoble (D. Dunand, in lit.). Pero en el herbario de Gay en Kew se conserva un pliego con un único ejemplar, que constituye un duplicado del material original en que Verlot basó A. villarsii, y que se toma como lectotipo de esta especie. Carece de sistema radical y posee varias hojas de hasta $50 \mathrm{~cm} \times 11 \mathrm{~mm}$, con la inflorescencia fragmentada simple, brácteas pardo-oscuras, cápsulas de 12-14 x 11-12 mm, ovoideoglobosas y pedicelos de 11 a $13 \mathrm{~mm}$, erectopatentes y ligeramente articulados por debajo de la mitad. Existe además en este pliego un sobre con dos flores de la misma procedencia, una con 12 tépalos y 12 estambres, enviadas a Gay también por Verlot el 18 de Mayo de 1856.

En este pliego de Kew se conserva un segundo sobre que contiene dos frutos y semillas de c. 8 × 3-4 mm, recibidos por Gay en Agosto de 1856 de un pliego existente en el herbario de Grenier, según Gay escribe sobre 


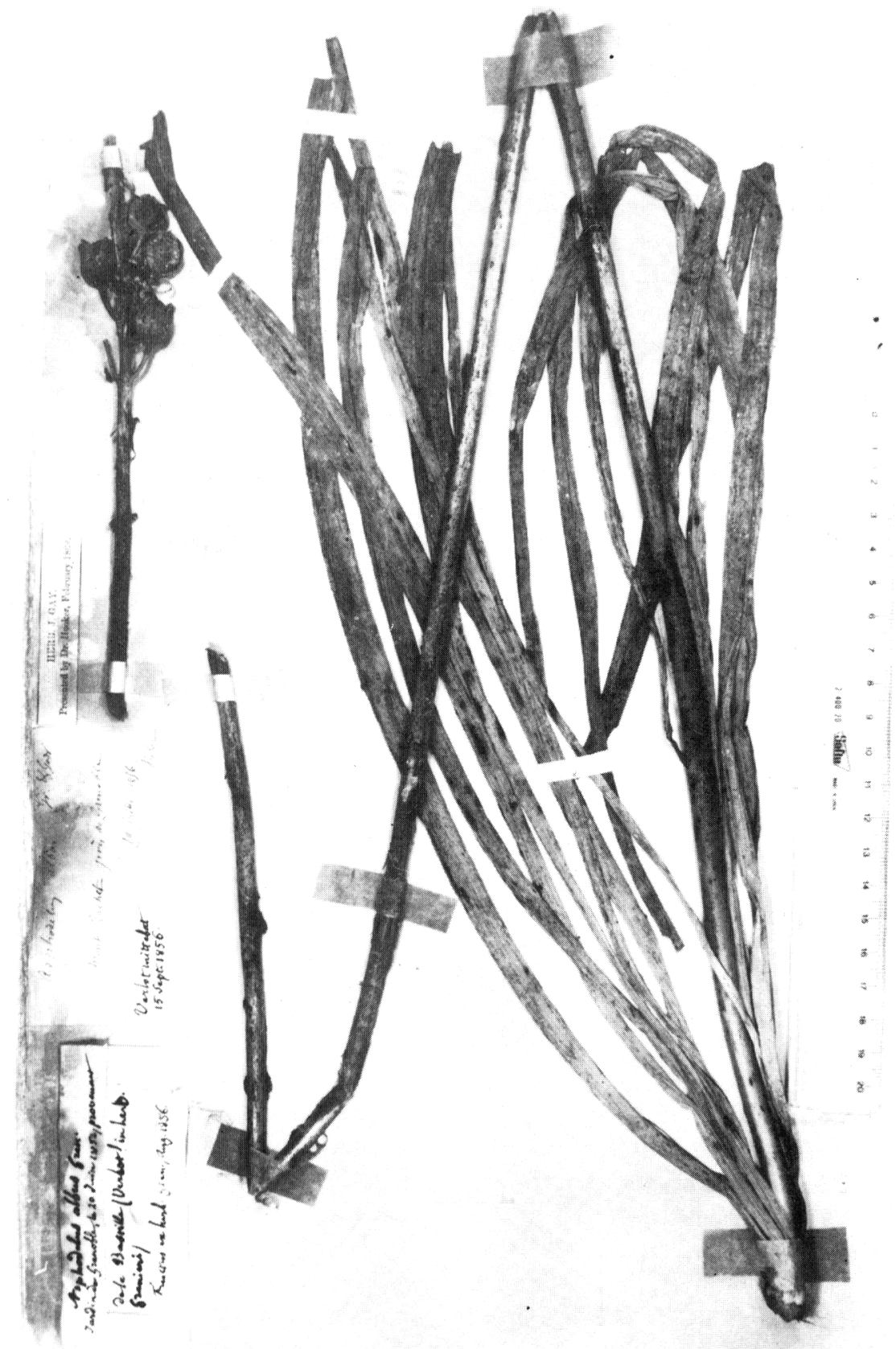

Figura 5. Lectotipo de Asphodelus villarsii Verlot (K). Lectotype of Asphodelus villarsii Verlot (K). 
este mismo sobre (fig. 4c). Se ha estudiado el pliego de Grenier, que se conserva en París y contiene un fragmento de un escapo con inflorescencia simple, densa, con brácteas oscuras, cápsulas de 14-15 x 12-13 mm, ovoideoglobosas sobre pedicelos erecto-patentes de 17-18 mm y 1-1,2 mm de grosor articulados hacia el 1/3 inferior, y semillas de 8,6 x 3,7 $\mathrm{mm}$. En la etiqueta derecha se puede leer la determinación manuscrita de Grenier como «Asphodelus villarsii Verlot», quien lo donó al Herbario del Museo de París en 1875. En la parte inferior izquierda hay una etiqueta en la que de forma manuscrita se indica: «Asphodelus ramosus L. / provenant de la Bastille / Jardin de Grenoble / 20 Juin 1852», lo que parece indicar que Verlot envió este pliego a Grenier. Verlot (1860: 148) comenta que había cultivado en el Jardín Botánico de Grenoble plantas recolectadas en Lautaret (Hautes-Alpes) y en las montañas calcáreas de los alrededores de Grenoble, y que había comunicado a Grenier en 1873 la existencia de dos tipos de plantas en el Delfinado.

Además, se ha estudiado otro pliego de K, que contiene en la mitad izquierda un ejemplar consistente en una roseta de varias hojas de hasta $40 \times 1,5 \mathrm{~cm}$ estrechadas hacia la base $\mathrm{y}$ rodeada en su base por algunas fibras finas $y$ un fragmento de escapo, además de dos inflorescencias simples sueltas, con las brácteas oscuras, pedicelos de 10-13 mm con la articulación poco marcada y situada por debajo de la mitad, y cápsulas globosas de 11 13 x 10-13 mm, con algunos tépalos persistentes. Este pliego perteneció al herbario de Gay, según se indica en una etiqueta del pliego: HERB. J. GAY / Presented by Dr. Hooker, February 1868 [impreso]. En la etiqueta de la parte inferior izquierda se lee: Asphodelus albus Gr. \& Godr. // Comboire près de Grenoble // 14 Juillet 1856 // Verlot [manuscr. Verlot] / / Verlot mittebat / 15 Sept. 1856 [manuscr. Gay]. Estos materiales constituyen paralectotipos de esta especie.

\section{Afinidades}

Por la información actual, parece evidente que ambos taxones han debido tener un antecesor común, a partir del cual se diferenciaron. A. villarsii quedó relegada a las partes altas de las montañas, adaptándose a un clima más frío y húmedo. A. cerasiferus quedó restringida a climas más mediterráneos, de carácter más xérico. Tanto una como la otra, pero sobre todo A. villarsii, muestran un marcado polimorfismo, el cual puede explicarse en gran parte como resultado de los mecanismos de especiación, que han sido favorecidos por el aislamiento geográfico al que han estado sometidas muchas de sus poblaciones.

No obstante, los procesos de hibridación han debido contribuir a un incremento en la variabilidad morfológica de ambos taxones. Sus áreas de distribución a menudo se ponen en contacto, y en tal caso, A. villarsii ocupa siempre las zonas más altas y $A$. cerasiferus las más bajas. Pero a veces coinciden en la misma localidad. Cuando esto ocurre, se ha comprobado la existencia de poblaciones hibridógenas en las que es difícil asignar las plantas a una u otra especie. No se ha comprobado experimentalmente su capacidad de hibridación pero cabe esperarla, si se tiene en cuenta el parecido morfológico entre ambas especies, la existencia en ambas de los mismos niveles de ploidía: diploide y tetraploide (Díaz Lifante, inédito), y la facilidad con la que se produce hibridación en la Sect. Asphodelus, incluso entre especies muy distantes morfológicamente.

En A. cerasiferus las poblaciones más típicas, en el sur de Francia y $\mathrm{NE}$ y $\mathrm{C}$ de España, se distinguen por su rizoma claramente horizontal y desprovisto de fibras o con algunas fibras delgadas, por sus brácteas siempre blanquecinas con nervio medio pardo, y por la parte basal de los filamentos estaminales marcadamente surcada en ambos verticilos y abruptamente atenuada. Las poblaciones 
costeras del E y $S$ de la Península Ibérica presentan rizoma generalmente vertical y provisto de abundantes fibras gruesas, sus brácteas son a veces de color pardo y la parte basal de los filamentos estaminales a menudo está surcada sólo en el verticilo externo.

En plantas de las sierras de Málaga, partes bajas de Sierra Bermeja y Montes de León, en el límite occidental de la distribución de esta especie, se presentan inflorescencias más densas de lo normal y brácteas más oscuras. En esta zona $A$. cerasiferus se pone en contacto con A. villarsii, siendo ésta frecuente en todas las partes altas de la Serranía de Ronda. En las sierras de la Cazorla, Guillimona, La Sagra y otras formaciones montañosas de las provincias de Jaén y Granada, que constituyen en el S de España el límite oriental de A. villarsii, esta especie coincide con $A$. cerasiferus en algunas zonas. La existencia de ejemplares en cierto modo intermedios entre estas dos especies, difíciles de asignar a una u otra, podrían explicarse de nuevo por la existencia de procesos de hibridación.

Asimismo, en el sur de Francia (departamentos de Var, Alpes Maritimes y Bouchesdu-Rhône), aparecen junto a ejemplares típicos de A. cerasiferus, con las brácteas pálidas, otros menos típicos, con brácteas pardo-oscuras, aunque éstas son cortas y estrechas, inflorescencias laxas y pedicelos más o menos largos. Muy posiblemente esta variabilidad debió ser la responsable de que Jordan (1860) y Jordan \& Fourreau (1868) describieran diversas especies en esta región, identificables con una u otra especie en gran parte de sus caracteres.

En cuanto a $A$. villarsii, la variabilidad que presenta esta especie a lo largo de su amplia área de distribución permite reconocer tres grupos infraespecíficos cuya delimitación y tratamiento se dará a conocer en una revisión taxonómica del género Asphodelus (Díaz Lifante y Valdés, inédito).

La existencia de hibridación entre $A$. cerasiferus y $A$. villarsii en las zonas en que coinciden sus áreas de distribución, unido al amplio polimorfismo que presenta $A$. villarsii, puede dificultar la delimitación de ambas especies. En el presente estudio se ponen de manifiesto las diferencias que permiten su reconocimiento a nivel específico. El estudio posterior detallado de los procesos de hibridación permitirá dilucidar más claramente la incidencia de dichos procesos en la variabilidad de ambas especies, así como sus consecuencias taxonómicas.

\section{BIBLIOGRAFÍA}

ARGÜELLES, J. M., J. DELGADO y M. LAÍNZ 1984- Contribuciones al conocimiento de la flora de Asturias, I. Bol. Cienc. Nat. I.D.E.A., 33: 3-14.

ASCHERSON, P. \& P. GRAEBNER -1905Synopsis der Mitteleuropäischen Flora, 3. Leipzig.

BILLOT, C. -1854- Flora Galliae et Germaniae Exsiccata, Herbier publié par C. Billot, Douzième, Treizième et Quartorzième Centuries. Archives de la Flore de la France et d'Allemagne, 1: 287-303.

BILLOT, C. -1857- Vingt-unième et vingt-deuxième centuries, Diciembre 1857. In: C. Billot (ed.), Annotations à la Flore de France et d'Allemagne. Haguenau.

COSTE, H. -1905- Flore descriptive et,ilustrée de la France, de la Corse et des Contrées limitrophes, 3. Paris.

GAY, J. E. -1857a- Sur la distribution géographique des trois espèces de la Section Gamon, du genre Asphodelus. Ann. Sci. Nat., Paris, 7: 116-134.

GAY, J. E. -1857b- Sur la distribution géographique des trois espèces de la Section Gamon du genre Asphodelus. Bull. Soc. Bot. France, 4: $607-$ 612 .

GAY, J. E. -1858- Société Botanique de France, séance du 14 Mai 1858. Bull. Soc. Bot. France, 5: 249-253.

JORDAN, A. -1860- Notice sur diverses espèces négligées du genre Asphodelus, comprises dans le type de l'Asphodelus ramosus de Linné. Bull. 
Soc. Bot. France, 7: 722-741.

JORDAN, A. \& J. FOURREAU -1868-Breviarium Plantarum Novarum, 2. Paris.

LEUSSINK, J. A. -1985- The publication dates of the Bulletin de la Société Botanique de France (vols. 1-25, 1854-1878). Taxon, 34: 583-606.

MAIRE, R. -1958-Flore de l'Afrique du Nord, 5. Paris.

MERINO, B. -1909- Flora descriptiva é ilustrada de Galicia, 3. Santiago.

MUTEL, A. -1830- Flore du Dauphiné, 1. Grenoble.

MUTEL, A. -1848- Flore du Dauphiné, ed. 2. Grenoble.

RICHARDSON, I. B. K. \& B. E. SMYTHIES 1978- Liliaceae, Asphodelus L. In: V. H. Heywood, Flora Europaea, Notulae Systematicae ad Floram Europaeam Spectantes, no. 20. Bot. J. Linn. Soc., 76: 368.
RICHARDSON, I. B. K. \& B. E. SMYTHIES 1980- Asphodelus L. In: T. G. Tutin et al. (eds.), Flora Europaea, 5: 17. Cambridge.

VERLOT, M. J. -1860-Sur l'Asphodelus ramosus de la Flore du Dauphiné de Villars. Bull. Soc. Statist. Dép. Isère, Sér. 2, 4: 146-155.

VILLARS, M. -1787- Histoire des Plantes de Dauphiné, 2. Grenoble.

WILLKOMM, M. -1862- Liliaceae. In: M. Willkomm \& J. Lange, Prodromus Florae Hispanicae, 1. Stuttgartiae.

Aceptado para su publicación en Julio de 1993

Dirección de los autores: Dpto. Biología Vegetal y Ecología, Facultad de Biología, Apdo. correos 1095 , 41080-Sevilla, España. 